\title{
Is it time to reassess alpha lipoic acid and niacinamide therapy in schizophrenia?
}

\author{
Sheila E.J. Seybolt * \\ Department of Kinesiology and Human Nutrition, University of Illinois at Chicago, 1919 Taylor, Chicago, IL 60612, United States \\ articleinfo \\ Article history: \\ Received 1 April 2010 \\ Accepted 15 July 2010 \\ Available online $x x x x$

\section{sum mary}

As sulfur containing thiols, alpha lipoic acid (ALA) and its reduced form dihydrolipoic acid (DHLA) are powerful antioxidants and free radical scavengers capable of performing many of the same functions as glutathione (GSH). ALA supplementation may help protect mitochondria from oxidative stress, a possible mechanism contributing to certain forms of brain diseases called schizophrenia. Shortly before the advent of antipsychotic medications, two small studies found ALA relieved psychiatric symptoms in schizophrenia. More recently, animal studies have shown ALA augmentation improves mitochondrial function. At pharmaceutical levels, niacinamide helps preserve mitochondrial membrane integrity and acts as an antioxidant. ALA is a precursor for lipoamide, an essential mitochondrial coenzyme and niacinamide is a component of niacinamide adenine dinucleotide (NAD). NADH, the reduced form of NAD, is involved in the reduction of ALA to DHLA within the mitochondria. This is relevant to contemporary research because DHLA increases GSH and low GSH levels contribute to mitochondrial dysfunction and oxidative stress which have been implicated in the pathophysiology of schizophrenia.

Introduction

Schizophrenia is a complex multifactorial disease of unknown etiology characterized by many structural and functional abnormalities, a partial list of which includes: mitochondrial dysfunction [1-3]; oxidative stress with the increased production of free radicals, reactive oxidative species (ROS) [4,5]; significant increases in nitric oxide synthase 3 (NOS3) in the prefrontal cortex (PFC) [3] and reactive nitrogen species (RNS) in the caudate [6] suggesting nitrosative stress; reduced antioxidant activity with lower levels of superoxide dismutase (SOD), GSH, glutathione peroxidase (GPx) [7], and ascorbic acid (vitamin C) [8,9]; deficiencies of mitochondrial electron transport chain (ETC) coenzyme Q10 (ubiquinone) [10]; higher levels of thiobarbituric acid reactive substances (TBARS) [11]; lower levels of membrane essential polyunsaturated fatty acids (PUFAS) [12]; oxidative damage to nerve and mitochondrial membranes [9]; dendritic spine abnormalities, including a reduction in their number and length with subsequent alterations in synaptic transmission and a decrease in the neuropile, as evidenced by electron microscope [13]; brain atrophy and ventricular enlargement [14]; disturbances of brain neurotransmitter activity including dopamine hyperactivity [15] and NMDA glutamate receptor hypofunction $[16,17]$.

In the treatment of schizophrenia the adjunctive use of relatively inexpensive supplements like antioxidant vitamins and niacinamide are staples in orthomolecular psychiatric practice [18] but are rarely used in mainstream psychiatry which continues to rely solely upon pharmaceuticals. This schism stems from a longstanding antagonism dating back to the 1960's [19] and the criticism that orthomolecular practitioners often failed to backup their claims with gold standard, double blind studies. Prabakaran's discovery that $49 \%$ of the differences between schizophrenic and normal brains are related to mitochondrial dysfunction and oxidative stress brings this issue to the table once again [3]. ALA, as lipoamide, is an essential mitochondrial coenzyme. As antioxidants, ALA and DHLA are potent scavengers of free radicals acting upon a number of ROS and RNS including singlet oxygen, superoxide radicals, hydrogen peroxide, hydroxyl radicals, hypochlorite, nitric oxide radicals, peroxynitrite and peroxyl radicals [20] many of which contribute to the oxidative stress of schizophrenia [6,9]. In the mitochondria, which lack catalase to remove toxic hydrogen peroxide $\left(\mathrm{H}_{2} \mathrm{O}_{2}\right), \mathrm{GSH}$ is used in the conversion of $\mathrm{H}_{2} \mathrm{O}_{2}$ to $\mathrm{H}_{2} \mathrm{O}$ and in the process $\mathrm{GSH}$ is oxidized to form glutathione disulfide (GSSG) [21]. A low GSH/GSSG ratio, an indicator of oxidative stress, is a characteristic of schizophrenia. Niacinamide is a component of NAD, NADH, NADP and NADPH. NADPH is used in the reduction of ALA to DHLA in the cytosol. DHLA helps maintain a favorable GSH/GSSG ratio by raising GSH through improved cysteine utilization [22] and by converting GSSG back to GSH [23].

\section{Hypothesis}

These data suggest the adjunctive use of ALA and niacinamide by reducing ROS and RNS, and improving the GSH/GSSG ratio may help alleviate mitochondrial dysfunction and oxidative stress and improve psychiatric symptoms in schizophrenia.

Testing this hypothesis would involve conducting a randomized, double-blind, placebo-controlled trial to determine what effect ALA/niacinamide therapy might have upon mitochondrial function, oxidative stress, GSH levels and clinical outcome. A potential protocol might include: Arm 1: starting with an antipsychotic and niacinamide in first week and adding ALA in the second week; Arm 2: starting with an antipsychotic and ALA in first week and adding placebo in the second week; Control: antipsychotic, with matched number of placebo pills for 4 weeks. While the concurrent use of antipsychotics prevents a direct comparison with earlier trials which used ALA as a monotherapy [24,25], not providing them would be medically unethical.

Schizophrenia affects approximately one out of a 100 individuals, of which about one third respond fully to treatment, one third incompletely and one third not at all. Part of the problem of inadequate response to therapy may be due to the fact that drugs in current use were not designed to treat GSH deficiency [26] or oxidative stress and the adjunctive use of antioxidants is not yet standard practice.

Evaluation of the hypothesis 
There is an increasing discussion about GSH deficiency as a major cause of the oxidative stress associated with damage to the schizophrenic brain $[13,14,17,27-29]$. While the mitochondrial ETC is highly efficient, some ROS 'leak' into the matrix. Since mitochondria do not contain catalase; they depend upon GSH peroxidase and non-enzymatic reaction with GSH to protect against ROS toxicity. However, mitochondria do not synthesize GSH, and must obtain their GSH from intracellular supplies [21,30].

GSH, a tripeptide of glutamate, glycine and cystine, does not cross the blood brain barrier (BBB) [29] but brain levels can be increased by GSH substrate supplementation [21] and the GSH/GSSG ratio improved [31]. ALA crosses the BBB and increases levels of GSH [20] by improving cystine utilization [22,31]. DHLA, the reduced form of ALA, is able to bypass the rate limiting cystine transport system by reducing cystine to cysteine which is then taken up by neutral amino acid transporters and used in the synthesis of GSH. In addition, DHLA is capable of directly reducing GSSG to GSH [23], thereby improving the GSH/GSSG ratio. GSH potentiates NMDA glutamate receptor function $[28,32]$ and scavenges peroxynitrite and superoxide anions, normally converted by SOD to hydrogen peroxide [9]. Supplementation with N-acetyl cystine, a GSH substrate, raises GSH levels in schizophrenia [33] but ALA with its unique antioxidant and free radical scavenging profile may be superior to $\mathrm{N}$-acetyl cystine as a means of raising $\mathrm{GSH}$ and combating mitochondrial dysfunction and oxidative stress.

The dopamine GSH connection

Dopamine reversibly inhibits mitochondrial complex I activity [1] and impairs mitochondrial membrane potential [34]. Mitochondria are particularly vulnerable to the presence of excess dopamine and its toxic metabolites because monamine oxidase (MAO), the enzyme responsible for dopamine metabolism, is located on the outer mitochondrial membrane [1]. Dopamine is a major source of ROS and when added to cultured cortical neurons in conditions of low GSH it further decreases GSH levels and reduces the number of neuronal processes (spines analogs) [13]. "The toxicity of the dopamine metabolites [in low GSH conditions] would be restricted to the microenvironment of the terminal of the dopamine fibers innervating the cortex. .. leading to the degeneration of spines and dendrites rather than of the entire cell body" [28]. If this is the mechanism behind the morphological changes in the brain observed by Grima and Buckman $[13,14]$ could the dendritic spine abnormalities and subsequent alterations in synaptic transmission be reversed and normal synaptic transmission restored? ALA has been reported to induce the sprouting of neurites in vitro $[20,35]$.

\section{Early studies of ALA in schizophrenia}

It has been over 50 years since two small clinical trials found low dose ALA monotherapy improved psychiatric symptoms in about half of the schizophrenics in which it was tried [24,25]. At the time ALA, also known as thioctic acid, was considered a vitamin with antioxidant properties. Giamattei, citing earlier workers' observations of liver abnormalities in schizophrenia, tested 27 male schizophrenics for liver dysfunction and from those, 10 patients who were most strongly positive on two tests for liver abnormalities were selected for ALA therapy. For 28 days they were given $10 \mathrm{mg}$ intravenous doses of ALA. In all 10 patients liver function normalized, with four showing appreciable improvement in their mental status and behavior and the remaining six unchanged. Ten days after discontinuation of ALA, tests for liver function were essentially the same with no mention of any change in psychic condition. In addition, Giamattei reported treating a newly diagnosed female schizophrenic with normal liver function, 2 months after the beginning of her symptoms, with daily doses of $10 \mathrm{mg}$ ALA given intravenously. By the 10th day there were signs of improvement in her condition and on the 15th day when the improvement was most evident electric shock therapy was begun and a little afterwards she was released from the hospital as improved [24].

The results of the second study are more difficult to interpret because the method of administration and the amounts of ALA given to each individual varied with no rationale for the discrepancies provided. Altschule who had previously shown that injected GSH improved keto-acid metabolism and mental status in a female paranoid schizophrenic [36], decided to study the effects of ALA as a "vitamin concerned with the utilization of keto acids". He chose six female chronic schizophrenics. Starting with intramuscular injections of ALA, daily doses ranged from 20 to $40 \mathrm{mg}$ increased to 100 to $200 \mathrm{mg}$ and oral doses starting at $100 \mathrm{mg}$ increased to100 mg two times a day. In general, smaller doses and doses given orally were better tolerated than larger doses or injections but the results varied from patient to patient with no consistency between the size of the initial dose or method of administration and outcome. Unlike the first study in which no patients experienced a worsening of psychiatric symptoms, two of the three patients who at first showed improvement at the lower dose, deteriorated when given a higher dose, the third was not given a higher dose. Another patient showed no change at the lower dose only to worsen when the dosage was increased. The remaining two experienced a worsening of symptoms at the onset of treatment and therapy was discontinued. Clinical worsening was accompanied by impairment of keto-acid utilization, marked by increases in lactate and pyruvate. This seems to correlate with the deficiencies in pyruvate dehydrogenase and elevated levels of lactate found in the schizophrenic prefrontal cortex by Prabakaran. Within 10 days of discontinuation of ALA therapy all patients returned to their previous psychiatric status [25].

Liver, heart and brain, all tissues with high metabolic rates, have high concentrations of mitochondria. If improvement in mitochondrial function was the basis for the reversal of liver malfunction in the Italian study, perhaps the demands of the liver reduced the availability of ALA to the brain, in effect, lowering the dose. The results of these two studies suggest a low dose therapeutic window and the possibility of a genetic basis for the variability in individual response, which in addition may be subject to modification by underlying pathological conditions like liver disease.

2

The ALA niacinamide connection

Niacinamide is a component of NAD and NADH. NADH, a substrate for the production of ATP, enters the ETC at mitochondrial Complex I. Mitochondrial Complex I activity is downregulated in schizophrenia $[1,3]$ and is deficient in mitochondrial encephalopathy, lactic acidosis and stroke-like episodes syndrome (MELAS). Oral niacinamide supplementation has been shown to raise blood NAD and reduce abnormal lactate and pyruvate blood levels in the treatment of MELAS, a genetically based condition characterized by mitochondrial Complex I deficiency, high lactate levels, reduced ATP production and oxidative stress where niacinamide in addition to its ability to reduce abnormal pyruvate and lactate levels by raising NAD/NADH was hypothesized to 
compensate for defective Complex I enzymes [37]. An increase in pyruvate and lactate was associated with the worsening of schizophrenic symptoms observed by Altschule [25] which suggests that niacinamide given prophylactically might be protective. Niacinamide, widely used in the orthomolecular treatment of schizophrenia $[38,39]$, is a precursor for NAD and NADH/NADPH. $\mathrm{NADH}$ produced in the Krebs cycle enters the ETC at mitochondrial Complex I where NADH dehydrogenase then transfers electrons from NADH to Coenzyme Q. The reduction in Complex I activity reported by Prabakaran "may be due to the depletion of reducing equivalents like NADH and NADPH which are utilized for the formation of GSH to counter oxidative damage to mitochondrial components" [40]. NADPH is the rate limiting factor in the regeneration of GSH during oxidative stress. As GSSG is reduced to GSH, NADPH is oxidized to NADP [41]. Niacinamide inhibits mitochondrial oxidative damage caused by ROS and acts as "an antioxidant capable of protecting cellular membranes in the brain" [42]. At high doses it is a free radical scavenger and prevents the intracellular depletion of NAD [43]. Niacinamide maintains mitochondrial membrane potential and prevents cytochrome $C$ release [44], the first step in the initiation of apoptosis. Could these observations help explain the mechanism behind the therapeutic effects of niacinamide in schizophrenia observed by Hoffer [38]? And if given prophylactically, would niacinamide prevent the increases in lactate and pyruvate associated with the worsening of psychiatric symptoms seen in the Altschule ALA trial?

ALA, as lipoamide, functions within the mitochondrial matrix as an essential coenzyme in pyruvate dehydrogenase and in the alpha keto-acid complexes, alpha ketoglutarate and alpha keto-acid dehydrogenase $[20,45]$. In the mitochondria, which lack catalase to remove toxic hydrogen peroxide $\left(\mathrm{H}_{2} \mathrm{O}_{2}\right), \mathrm{GSH}$ is used in the conversion of $\mathrm{H}_{2} \mathrm{O}_{2}$ to $\mathrm{H}_{2} \mathrm{O}$ and in the process $\mathrm{GSH}$ is oxidized to form glutathione disulfide (GSSG) [21]. A low GSH/GSSG ratio, an indicator of oxidative stress, is characteristic of schizophrenia. ALA, commonly sold as a nutritional supplement, is a 50/50 mix of two biologically active enantiomers [20], a naturally occurring R-form and an S-form, with the R-form being the preferred substrate for reduction to DHLA by dihydrolipamide dehydrogenase using NADH in the mitochondria. The S-form is the preferred substrate for the reduction to DHLA by glutathione reductase using NADPH in the cytosol $[46,45]$. This suggests the co-administration of ALA/niacinamide might be synergistic, increasing the availability of DHLA by raising NADH and NADPH levels. DHLA in turn would help raise GSH by improving the utilization of its substrate cysteine [22] and by reducing GSSG back to GSH [23].

\section{Discussion}

Altschule [25] reported problems using relatively small amounts of ALA whereas a recent Korean study investigating ALA in the treatment of antipsychotic drug-induced weight gain in patients with schizophrenia used $1200 \mathrm{mg} /$ day orally [47], six times as much as Altschule's largest dose without major difficulties. This suggests the concurrent use of atypical antipsychotics may be protective and large doses of ALA safer than expected but are doses at this level still therapeutic? Of the seven patients studied, three showed no change in psychiatric symptoms, two patients were dropped from the study because they stopped taking their antipsychotics and experienced an increase in depressive symptoms necessitating a change in medication and two showed modest improvement. One reported some increase in volition for daily living. The other seemed to talk more than before in group therapy (Hyun-Sang Cho, personal communication).

ALA improves cognition in aged mice [48] and mitochondrial function in aged rats [49-51]. It has shown promise in treating ischemia-reperfusion. Interestingly, both Cardoso do Vale [52] and Schonheit [53] reported a dose response threshold for ALA similar to the therapeutic window noted above in the Giamattei and Altschule trials. Cardoso do Vale found 6.06 mM ALA to be the most therapeutic dose but concluded that at both lower $(3.03 \mathrm{mM})$ and higher $(60.6 \mathrm{mM})$ levels ALA had a pro-oxidant effect. Pro-oxidant activity for ALA has also been reported by Moini [23]. Schonheit found $0.5 \mathrm{lM}$ doses of ALA beneficial but harmful when increased to $10 \mathrm{lM}$. Does the common low dose therapeutic window for ALA provide evidence for a relationship between ischemia reperfusion and schizophrenia and does this in turn support the contention that schizophrenia is in part a disease of chronic, intermittent hypoxia and ischemia with possible microvasculature abnormalities in the prefrontal cortex as Prabakaran has proposed?

\section{Looking for a genetic connection}

Two enzymes are involved in the synthesis of GSH: glutathione synthetase (GSS) and gamma glutamyl cysteine synthetase, also known as glutamate cysteine ligase $(G C L)$ with $G C L$ being the rate-limiting enzyme and cysteine the rate-limiting substrate. $G C L$ is composed of two subunits: GCL modifier (GCLM) and GCL catalytic subunit (GCLC). Recent evidence suggests a possible genetic link to schizophrenia with GCLM being identified as a susceptibility gene [54]. But so far no one gene or combination of genes has been identified as a cause of schizophrenia and there are many competing alternative models in contention with no consensus in sight. We are still far from a complete understanding of the pathophysiology of schizophrenia [55]. Efforts to explain its etiology are reminiscent of the story of the three blind men attempting to describe an elephant with each holding firmly to a different part of the animal. Each is correct in their own way but fall short because they cannot see the big picture. In schizophrenia research this problem is compounded by the possibility that there may be more than one species of elephant in the room. . the schizophrenias.

This hypothesis is not an attempt to advance a new causal theory for schizophrenia or to defend an existing one but is an effort to find a solution to the problem of incomplete response to current therapy in the hope that the adjunctive use of ALA will help relieve symptoms and restore functioning, if not for all, at least for some. Subsequently, if treatment responders can be differentiated genetically, then using this backdoor approach may help clarify the etiology and lead to the development of new and better treatments. Whether ALA combined with antipsychotics, with or without the addition of niacinamide, can be beneficial to an equal or greater percentage of patients than was previously shown by Giamattei and Altschule remains to be discovered.

\section{Conflicts of interest statement}

None declared.

Acknowledgement

The author would like to thank Ms. Maria Abbinanti for her assistance in translating the Giamattei paper and Dr. Dennis Beedle 
for reading this manuscript. His critical comments and encouragement were invaluable. This work was unfunded.

\section{References}

[1] Ben-Shachar D. Mitochondrial dysfunction in schizophrenia: a possible linkage to dopamine. J Neurochem 2002;83:1241-51.

[2] Karry R, Klein E, Ben-Shachar D. Mitochondrial complex I subunits expression is altered in schizophrenia: a postmortem study. Biol Psychiatry

2004;55:676-84.

[3] Prabakaran S, Swatton JE, Huffaker SJ, et al. Mitochondrial dysfunction in schizophrenia: evidence for compromised brain metabolism and oxidative stress. Mol Psychiatry 2004;9:684-97.

[4] Mahadik SP, Mukherjee S. Free radical pathology and antioxidant defense in schizophrenia - a review. Schizophr Res 1996;19:1-7.

[5] Reddy RD, Yao JK. Free radical pathology in schizophrenia - a review.

Prostaglandins Leukot Essent Fatty Acids 1996;55:33-43.

[6] Yao JK, Leonard S, Reddy RD. Increased nitric oxide radicals in postmortem

brain from patients with schizophrenia. Schizophr Bull 2004;30:923-34.

[7] Yao JK, Leonard S, Reddy R. Altered glutathione redox state in schizophrenia. Dis Markers 2006;22:83-93.

[8] Suboticanec K, Folnegovic-Smalc V, Korbar M. Vitamin C status in chronic schizophrenia. Biol Psychiatry 1990;28:959-66.

[9] Yao JK, Reddy RD, Van Kammen DP. Oxidative damage in schizophrenia an overview of the evidence and its therapeutic implications. CNS Drugs 2001;15:287-310

[10] Imagawa M. Low erythrocyte coenzyme Q10 in schizophrenic patients. Jpn J Psychiatry Neurol 1989;43:143-5.

[11] Mahadik SP, Mukherjee S, Scheffer R, Correnti EE, Mahadik JS. Elevated Plasma lipid peroxides at the onset of nonaffective psychosis. Biol Psychiatry 1998;43:674-9.

[12] Yao JK, Leonard S, Reddy RD. Membrane phospholipid abnormalities in postmortem brains from schizophrenic patients. Schizophr Res 2000;42:7-17. [13] Grima G, Benz B, Parpura V, Cuenod M, Do KQ. Dopamine-induced oxidative stress in neurons with glutathione deficit: implication for schizophrenia. Schizophr Res 2003;62:213-24.

[14] Buckman TD, Kling AS, Sutphin MS, Steinberg A, Eiduson S. Platelet glutathione peroxidase and monamine oxidase activities in schizophrenics with CT scan abnormalities: relation to psychosocial variables. Psychiatry Res

1990;31:1-14.

[15] Abi-Dargham A. Do we still believe in the dopamine hypothesis? New data bring new evidence. Int J Neuropsychopharmacol 2004;Suppl.1:S1-5.

[16] Heresco-Levy U, Javitt DC. Comparative effects of glycine and D-cycloserine on persistent negative symptoms in schizophrenia: a retrospective analysis.

Schizophr Res 2004;66:89-96.

[17] Steullet P, Neijt JT, Cuenod M, Do KQ. Synaptic plasticity impairment and hypofunction of NMDA receptors induced by glutathione deficit: relevance to schizophrenia. Neuroscience 2006;137:807-19.

[18] Edelman E. Natural healing for schizophrenia and other common mental disorders. 3rd ed. Borage Books; 2001.

[19] Hoffer LJ. Vitamin therapy in schizophrenia. Isr J Psychiatry Relat Sci 2008;45(1):3-10.

[20] Packer L, Tritschler HJ, Wessel K. Neuroprotection by the metabolic antioxidant alpha-lipoic acid. Free Radic Biol Med 1997;22(1-2):359-78.

[21] Anderson ME. Glutathione: an overview of biosynthesis and modulation. Chem Biol Interact 1998;111-112:1-14.

[22] Han D, Handelman G, Marcocci L, Sen CK, Roy S, Kobuchi H, et al. Lipoic acid improves de novo synthesis of glutathione by improving cystine utilization.

Biofactors 1997;6:321-8.

[23] Moini H, Packer L, Saris NL. Antioxidant and prooxidant activities of alpha lipoic acid and dihydrolipoic acid. Toxicol Appl Pharmacol 2002;182:84-90. [24] Giamattei L. Thioctic acid in therapy of schizophrenia. Osp Psichiatr 1957;25:221-8.

[25] Altschule MD, Goncz RM, Holliday PD. Carbohydrate metabolism in brain disease. XI. Effects of thioctic (alpha-lipoic) acid in chronic schizophrenia. AMA Arch Intern Med 1959;103:726-9.

[26] Carter CJ. Schizophrenia susceptibility genes converge on interlinked pathways related to glutamatergic transmission and long-term potentiation, oxidative stress and oligodendrocyte viability. Schizophr Res 2006;86:1-14.

[27] Castagne V, Cuenod M, Do KQ. An animal model with relevance to schizophrenia: sex-dependant cognitive deficits in osteogenic disorderShionogi rats induced by glutathione synthesis and dopamine uptake inhibition during development. Neuroscience 2004;123:821-34.

[28] Do KQ, Trabesinger AH, Kirsten-Kruger M, Lauer CJ, Dydak U, Hell D, et al. Schizophrenia: glutathione deficit in cerebrospinal fluid and prefrontal cortex in vivo. Eur J Neurosci 2000;12:3721-8.

[29] Jain A, Martensson J, Stole E, Auld PA, Meister A. Glutathione deficiency leads to mitochondrial damage in the brain. Proc Natl Acad Sci USA

1991;88:1913-7.

[30] Griffith OW, Meister A. Origin and turnover of mitochondrial glutathione. Proc

Natl Acad Sci USA 1985;82:4668-72.

[31] Suh JH, Wang H, Liu RM, Liu J, Hagen TM. (R)-alpha lipoic acid reverses the agerelated loss in GSH redox status in post-mitotic tissues: evidence for increased

cysteine requirement for GSH synthesis. Arch Biochem Biophys

2004;423:126-35.

[32] Oja SS, Janaky R, Varga V, Saransaari P. Modulation of glutamate receptor 
functions by glutathione. Neurochem Int 2000;37:299-306.

[33] Berk M, Copolov D, Dean O, Lu K, Jeavons S, Schapkaitz I. N-acetyl cysteine as a glutathione precursor for schizophrenia - a double-blind, randomized,

placebo-controlled trial. Biol Psychiatry 2008;64(5):361-8 [Epub 2008 April

23].

[34] Elkashef AM, Al-Barazi H, Venable D, et al. Dopamine effect on the mitochondrial potential of B lymphocytes of schizophrenic patients and

normal controls. Prog Neuropsychopharmaco Biol Psychiatry 2002;26:145-8.

[35] Dimpfel W, Spuler M, Pierau FK, Ulrich H. Thioctic acid induces dosedependent sprouting of neurites in cultured rat neuroblastoma cells. Dev

Pharmacol Ther 1990;14:193-9.

[36] Altschule MD, Goncz RM, Henneman DH, Holliday PD. Carbohydrate

metabolism in brain disease. VII. The effect of glutathione on carbohydrate

intermediary metabolism in schizophrenia and manic-depressive psychoses.

AMA Arch Intern Med 1957;99:22-7.

[37] Majamaa K, Rusanen H, Remes A, Hassinen IE. Metabolic interventions against complex I deficiency in MELAS syndrome. Mol Cell Biochem 1997;174:291-6.

[38] Hoffer A. Use of nicotinic acid and/or nicotinamide in high doses to treat schizophrenia. Can J Psychiatry Nurs 1966;7:5-6.

[39] Klaidman LK, Mukherjee SK, Adams Jr JD. Oxidative changes in brain pyridine nucleotides and neuroprotection using nicotinamide. Biochim Biophys Acta 2001;1525(1-2):136-48.

[40] Mythili Y, Sudharsan PT, Varalakshmi PT. DL alpha lipoic acid ameliorates cyclophosphamide induced cardiac mitochondrial injury. Toxicology 2005;215:108-14

[41] Adams Jr JD, Klaidman LK, Chang ML, Yang J. Brain oxidative stress-analytical chemistry and thermodynamics of glutathione and NADPH. Curr Top Med

Chem 2001;1(6):473-82.

[42] Kamat JP, Devasagayam TP. Nicotinamide (vitamin B3) as an effective antioxidant against oxidative damage in rat brain mitochondria. Redox Rep 1999;4:179-84.

[43] Gale EA. Theory and practice of nicotinamide trials in pre-type 1 diabetes. J

Pediatr Endocrinol Metab 1996:9:375-9.

[44] Chong ZZ, Lin SH, Maiese K. Nicotinamide modulates mitochondrial

membrane potential and cysteine protease activity during cerebral vascular endothelial cell injury. J Vasc Res 2002;39:131-47.

[45] Packer L, Tritschler HJ. Alpha-lipoic acid: the metabolic antioxidant. Free Radic

Biol Med 1996;20:625-6.

[46] Haramaki N, Han D, Handelman GJ, Tritschler HJ, Packer L. Cytosolic and

mitochondrial systems for NADH-and NADPH-dependent reduction of alphalipoic acid. Free Radic Biol Med 1997;22:535-42.

[47] Kim E, Park DW, Choi SH, Kim JJ, Cho HS. A preliminary investigation of alphalipoic acid treatment of antipsychotic drug-induced weight gain in patients

with schizophrenia. J Clin Psychopharmacol 2008;28(2):138-46.

[48] Stoll S, Hartmann H, Cohen SA, Muller WE. The potent free radical scavenger

alpha-lipoic acid improves memory in aged mice. Putative relationship to

NMDA receptor deficits. Pharmacol Biochem Behav 1993;46:799-805.

[49] Arivazhagan P, Ramanathan K, Panneerselvam C. Effect of DL-alpha-lipoic acid on mitochondrial enzymes in aged rats. Chem Biol Interact 2001:138:189-98.

[50] Arivazhagen P, Shila S, Kumaran S, Panneerselvam C. Effect of DL-alpha-lipoic acid on the status of lipid peroxidation and antioxidant enzymes in various

brain regions of aged rats. Exp Gerontol 2002;37:803-11.

[51] Hagen TM, Liu J, Lykkesfeldt J, et al. Feeding acetyl L-carnitine and lipoic acid to

old rats significantly improves metabolic function while decreasing oxidative

stress. Proc Natl Acad Sci USA 2002;99:1870-5.

[52] Cardoso do Vale O, Fonteles DSR, Cabral FR, Fonteles MC. A dual action of alipoic

acid in the brain: an electrophysiological evaluation. Arq Neuropsiquiatr

2003:61:738-45.

[53] Schonheit K, Gille L, Nohl H. Effect of alpha-lipoic acid and dihydrolipoic acid

on ischemia/reperfusion injury of the heart and heart mitochondria. Biochim

Biophys Acta 1995;1271:335-42.

[54] Tosic M, Ott J, Barral S, et al. Schizophrenia and oxidative stress: glutamate

cysteine ligase modifier as a susceptibility gene. Am J Hum Genet

2006;79:586-92.

[55] Lakhan SE, Vieira KF. Schizophrenia pathophysiology: are we any closer to a

complete model? Ann Gen Psychiatry 2009;8:12.

S.E.J. Seybolt / Medical Hypotheses xxx (2010) xxx-xxx 3

0306-9877/\$ - see front matter_2010 Elsevier Ltd. All rights reserved.

doi:10.1016/j.mehy.2010.07.034

* Address: 7635 Jackson Blvd., Forest Park, IL 60130, United States. Tel.: +1 708

3660049.

E-mail addresses: sejs@att.net, sseybo2@uic.edu

Medical Hypotheses xxx (2010) xxx-xxx

Contents lists available at ScienceDirect

\section{Medical Hypotheses}

journal homepage: www.elsevier.com/locate/mehy

Please cite this article in press as: Seybolt SEJ. Is it time to reassess alpha lipoic acid and niacinamide therapy in schizophrenia?. Med Hypotheses (2010),

doi:10.1016/j.mehy.2010.07.034 